\title{
Prognosis of Full-Thickness Skin Defects in Premature Infants
}

\author{
Hyung Suk Moon, Jin Sik Burm, Won Yong Yang, Sang Yoon Kang \\ Department of Plastic and Reconstructive Surgery, Kyung Hee University School of Medicine, Seoul, Korea
}

Background In the extremities of premature infants, the skin and subcutaneous tissue are very pliable due to immaturity and have a greater degree of skin laxity and mobility. Thus, we can expect wounds to heal rapidly by wound contraction. This study investigates wound healing of full-thickness defects in premature infant extremities.

Methods The study consisted of 13 premature infants who had a total of 14 cases of fullthickness skin defects of the extremities due to extravasation after total parenteral nutrition. The wound was managed with intensive moist dressings with antibiotic and anti-inflammatory agents. After wound closure, moisturization and mild compression were performed.

Results Most of the full-thickness defects in the premature infants were closed by wound contraction without granulation tissue formation on the wound bed. The defects resulted in 3 pinpoint scars, 9 linear scars, and 2 round hypertrophic scars. The wounds with less granulation tissue were healed by contraction and resulted in linear scars parallel to the relaxed skin tension line. The wounds with more granulation tissue resulted in round scars. There was mild contracture without functional abnormality in 3 cases with a defect over two thirds of the longitudinal length of the dorsum of the hand or foot. The patients' parents were satisfied with the outcomes in 12 of 14 cases.

Conclusions Full-thickness skin defects in premature infants typically heal by wound contraction with minimal granulation tissue and scar formation probably due to excellent skin mobility.

Keywords Premature infants / Skin defects / Wound healing / Cicatrix
Correspondence: Jin Sik Burm

Department of Plastic and Reconstructive Surgery, Kyung Hee University School of Medicine, 23 Kyungheedae-ro, Dongdaemun-gu, Seoul 130-872, Korea

Tel: +82-2-958-8431

Fax: +82-2-963-5638

E-mail: jsburm@gmail.com
This article was presented at the 1 st Research and Reconstructive Forum on May 12-13, 2011 in Deajeon, Korea.

No potential conflict of interest relevant to this article was reported.

Received: 8 May 2012 • Revised: 10 Jul 2012 • Accepted: 11 Jul 2012

pISSN: 2234-6163 • eISSN: 2234-6171 • http://dx.doi.org/10.5999/aps.2012.39.5.463 • Arch Plast Surg 2012;39:463-468

\section{INTRODUCTION}

Premature infants are live-born babies delivered before 37 complete weeks of gestation $[1,2]$. Their skin is still thin, reddish, and wrinkled with a small amount of subcutaneous fat due to immaturity [1]. Thus, the skin is softer and more pliable, resulting in a greater degree of skin laxity and mobility than a full-term infant has [2]. Full-thickness skin defects usually heal by such processes as granulation tissue formation, wound contraction, and marginal epithelialization [3-5]. Wound contraction reduces the size of the defect without the formation of new granulation tissues and accelerates the healing process better than epithelialization or scar formation does. The regional differences in wound contraction are probably due to relative differences in skin laxity [5]. We hypothesizedhat skin wounds in premature infants would close rapidly with less scar formation than in adults, and information 
about their wound healing could be applied to general wound management. The purpose of this paper is to investigate the prognosis of full-thickness defects in premature infants' extremities and their clinical significance.

\section{METHODS}

The current study consisted of 13 premature infants (14 cases) with full-thickness skin defects due to extravasation of total parenteral nutrition (Table 1). The gestational ages at birth ranged from 28 weeks and 4 days to 35 weeks and 6 days. The timing of extravasation was 14 to 50 days after birth.

Local and systemic treatments were performed after the infants were referred from the Department of Pediatrics. For local treatment, conservative management was performed and the defects healed by secondary intention. After cleansing the wound with normal saline, a sufficient amount of an antibiotic and anti-inflammatory ointment mixture was topically applied to the whole region of the wound site, followed by a saline-moistened gauze dressing. This treatment was performed twice a day during the acute phase and once a day during the convalescent phase. The necrotic tissue was removed when it was clearly demarcated. For systemic treatment, we administered prophylactic antibiotics during the acute stage and ascorbic acid mixed with fluids at a daily dose of 50 to $120 \mathrm{mg}$ as the antioxidants. After wound closure, topical oil moisturization and mild compression were applied until the scars were stabilized.

We reviewed the size of the defect, duration of closure, healing pattern, and shape of the scar from the medical records and photographs. At a final follow-up visit, we evaluated the degree of the parents' satisfaction with the treatment outcomes by showing photographs of the initial defects and the final scar. The degree of satisfaction was graded at one of four levels: excellent, good, fair, and poor.

\section{RESULTS}

Among all of the 14 cases of full-thickness defects in the 13 premature infants, the defects were present on the hand or the wrist in 5 cases, on the foot or the ankle in 8 cases, and on the elbow in 1 case. The size of the defects ranged from $0.5 \mathrm{~cm}$ to threefourths of the longitudinal length of the dorsum of the hand or foot. The defects had completely closed 14 to 55 days after injury. The follow-up periods ranged from 3 to 41 months (Table 1).

The vast majority of the defects closed by wound contraction. The direction of wound contraction was usually parallel to the longitudinal axis of the extremity, that is, vertical to the relaxed skin tension line (RSTL). The geometric shape of the defect gradually changed to elliptical. The shapes of the scars were pinpoint in 3 cases, linear in 9 cases, and round in 2 cases.

The defects smaller than $1 \mathrm{~cm}^{2}$ healed by wound contraction without granulation tissue formation and resulted in pinpoint scars (Fig. 1). In the linear scar cases, the defects were healed by wound contraction with no or minimal granulation tissue formation. In cases with no granulation tissue, the wound was covered using a whitish devitalized patch until wound closure. Minimal granulation tissue did not adhere to the marginal skin and slightly protruded with a shiny surface similar to one in a chronic wound of a pressure sore, and wound contraction readily occurred. Additionally, marginal epithelialization was extremely minimal. The linear scars were always parallel to the RSTL and rapidly transformed into mature scars (Fig. 2).

Two cases resulted in a round scar (Fig. 3). In these cases, initial wound care was delayed or intensive management was ne-

Table 1. Summary of full-thickness skin defects due to extravasation injury in 13 preterm infants

\begin{tabular}{|c|c|c|c|c|c|c|c|}
\hline Case & GP (wk) & Age (day) & Defect site & Size $(\mathrm{cm})$ & Closure (day) & Outcome & Satisfaction degree \\
\hline 1 & 29 & 50 & Rt hand dorsum & $2 / 3^{\text {a) }}$ & 35 & $\mathrm{RH}$, contr & Fair \\
\hline 2 & 33 & 18 & Lt wrist & $1 \times 0.8$ & 41 & PP & Excellent \\
\hline 3 & 29 & 42 & Lt foot dorsum & $2 / 5^{b)}$ & 50 & L & Excellent \\
\hline 4 & 28 & 19 & Rt foot, medial & $2 \times 0.7$ & 14 & $L$, fine & Excellent \\
\hline 5 & 28 & 32 & Rt ankle, lateral & $2.5 \times 2.5$ & 35 & L & Excellent \\
\hline 6 & 35 & 17 & Lt foot, lateral & $1.5 \times 1.5$ & 22 & $L$, fine & Excellent \\
\hline 7 & 29 & 11 & Lt elbow, anterior & $0.5 \times 0.5$ & 15 & PP & Excellent \\
\hline 8 & 32 & 14 & Rt ankle, medial & $2 \times 2$ & 38 & L & Good \\
\hline 9 & 34 & 22 & Rt foot, medial & $3 / 5^{b)}$ & 28 & $L$, fine & Excellent \\
\hline 10 & 30 & 28 & Rt foot dorsum & $1 / 2^{b)}$ & 36 & L & Excellent \\
\hline 11 & 29 & 22 & Lt hand dorsum & $1 / 2^{a)}$ & 27 & $\mathrm{RH}$ & Fair \\
\hline 12 & 31 & 10 & Lt foot dorsum & $3 / 4^{\text {b) }}$ & 55 & $\mathrm{~L}$, contr & Good \\
\hline 13 & 29 & 25 & Rt hand dorsum & $0.5 \times 0.5$ & 14 & PP & Excellent \\
\hline 14 & 33 & 22 & Rt hand dorsum & $3 / 4^{a)}$ & 43 & $\mathrm{~L}$, contr & Good \\
\hline
\end{tabular}


Fig. 1. A case of a pinpoint scar

(A) Full-thickness skin defect 34 days after extravasation injury. (B) Eighteen months after treatment.
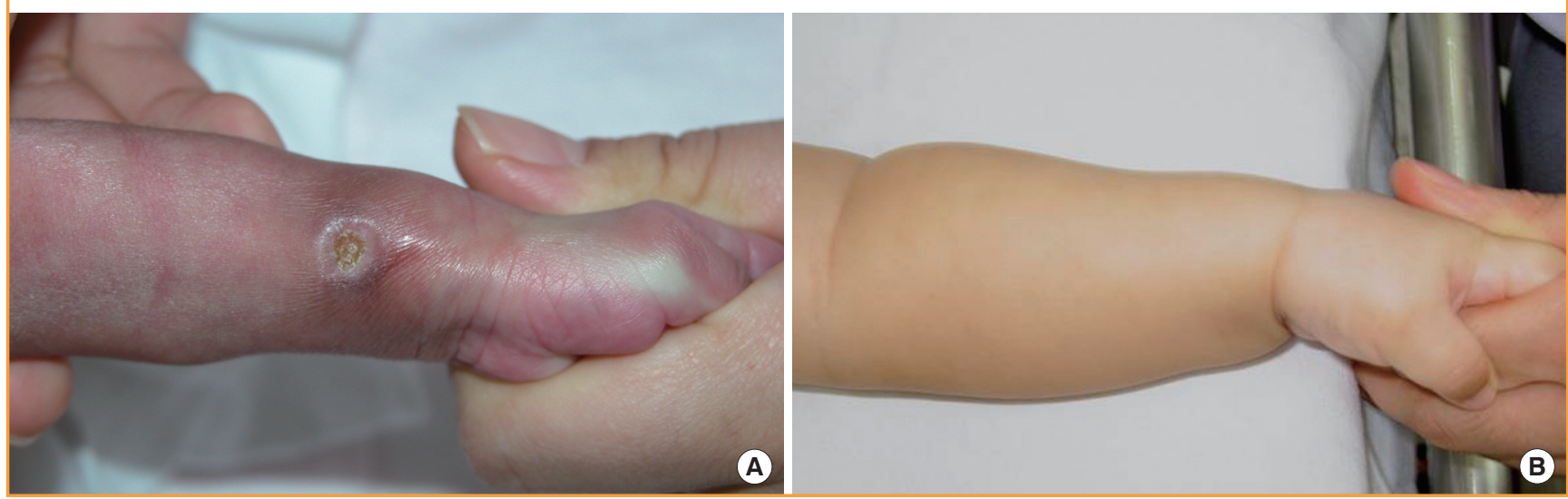

\section{Fig. 2. The cases of linear scars}

(A) Extravasation on the left dorsum of the foot. A full-thickness skin defect was noted 18 days later. (B) Three months after treatment, there was a linear scar without contracture. (C) A full-thickness skin defect of three-fourths of the hand dorsum in longitudinal length. (D) Five months after treatment, a linear scar with mild contracture was observed, but no functional deformity remained.
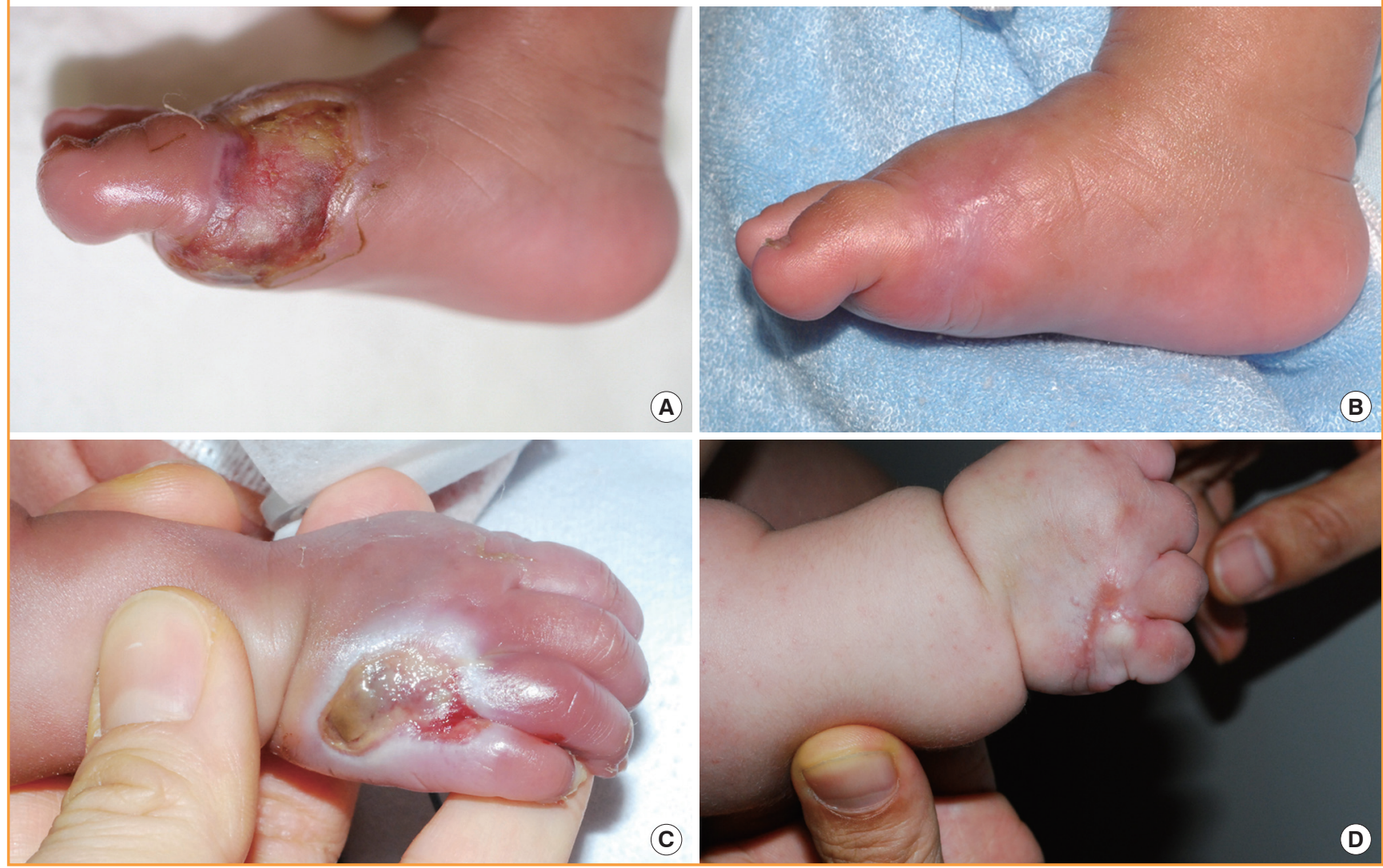

glected. During wound contraction, beefy-red granulation tissue ally converted to slightly thick scars.

appeared on the wound bed and adhered to the marginal skin. Consequently, it was covered by marginal epithelialization. After wound closure, the round scars became gradually erythematous and slightly hypertrophic. The hypertrophic scars improved after intralesional steroid injection and compression, and eventu-

Mild contracture due to wound contraction was observed in 2 cases with linear scars on the dorsum of the hand and foot (Fig. $2 \mathrm{C}, \mathrm{D})$ and one case with a round scar on the dorsum of the hand (Fig. 3A, B). All of these occurred in defects that were over two-thirds of the longitudinal length of the dorsum of the hand 


\section{Fig. 3. A case of a round hypertrophic scar with mild contracture}

(A) A full-thickness defect of two-thirds of the right hand dorsum in longitudinal length 18 days after extravasation injury. (B) Ten weeks after treatment.

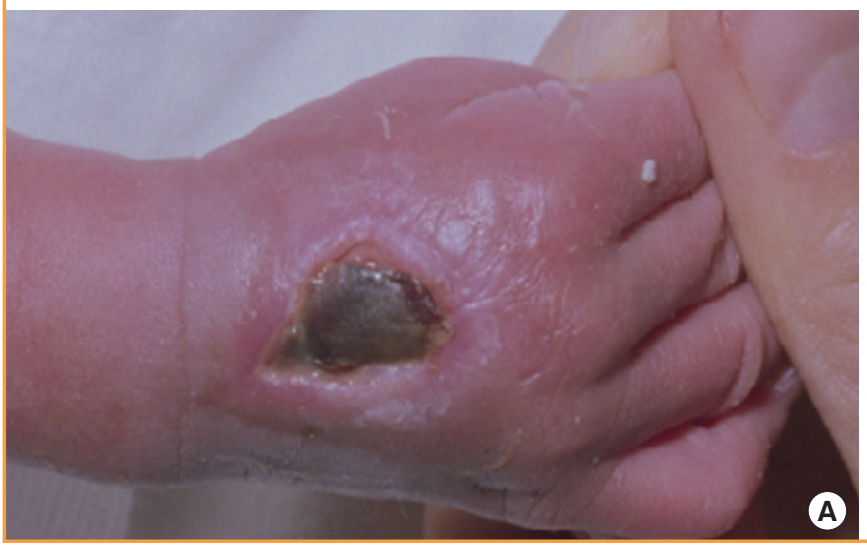

or foot. Despite the contracture, there was no functional limitation of motion of the fingers or toes.

The degree of the parents' satisfaction was excellent in 9 cases of pinpoint scars and linear scars, good in 3 cases of depressed or mild contracted linear scars, fair in 2 cases of round hypertrophic scars, and poor in 0 cases.

\section{DISCUSSION}

Cutaneous closed wounds heal without scar formation in the early gestational fetus $[5-8]$. The fetal skin grafts heal wounds without scarring after transplantation to the subcutaneous tissue (internal environment) of adult mice [9]. When the adult skin is transplanted onto a fetus, the adult skin heals with scar formation and fetal skin heals without scarring [10]. Thus, it has been persistently argued that amniotic fluid (external environment) and fetal serum (internal environment) are not required for scarless healing $[5,9]$, and scarless repair appears to be inherent to the fetal tissue and probably depends on factors associated with skin development [5].

Premature infants, delivered before 37 completed weeks of gestation, have premature skin (red, thin, smooth, shiny, and wrinkled with lanugo), less subcutaneous fat, and lower muscle tone than a full-term infant $[1,2]$. Thus, their skin is also very soft and pliable and has a high degree of skin laxity and mobility than a full-term infant. Additionally, the skin has a functionally immature epidermal barrier with significant transepidermal water loss $[2,11,12]$. The maturation of the skin barrier for functional adaptation to the extrauterine environment is fulfilled in 2 to 4 weeks, but dermal microcirculation continues to develop even beyond the neonatal period [13]. This may cause dry skin, vulnerability to trauma, and rapid onset of microbial colonization. Due to this skin immaturity, special skin care for moistur-

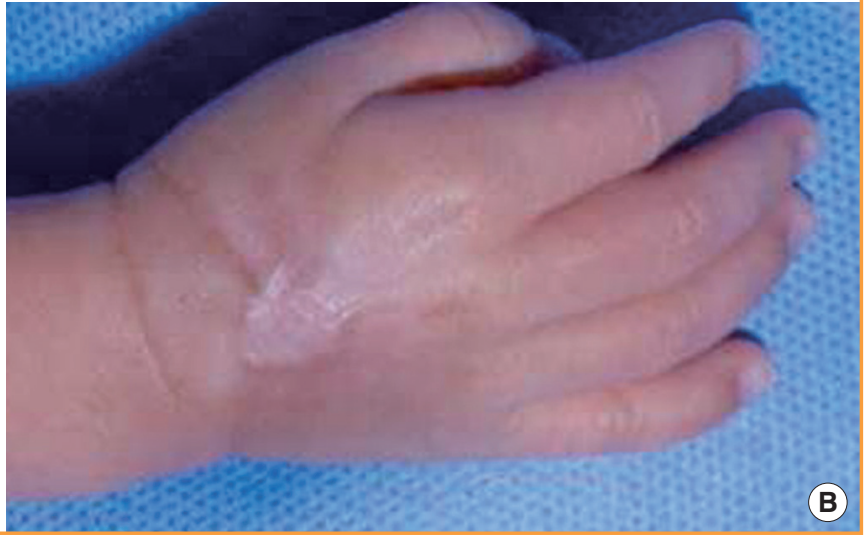

ization (external environment) is required for wound healing and epidermal barrier repair [11].

In the current study, wound healing of the full-thickness skin defects due to extravasation injury in the premature infant extremities usually seemed to have several characteristics of less inflammatory response, less granulation tissue formation, maximum wound contraction, minimal epithelialization, and consequently minimal scar formation.

Wound contraction is a process in which the surrounding skin is pulled circumferentially toward an open wound [1-3]. This process is an important part of wound healing because there is a dramatic decrease in the defect size without new tissue formation. It accelerates wound closure more than epithelialization itself and makes the scar size smaller. The amount of contraction is related to the wound size and skin mobility [3]. Animals have a much greater capacity for wound contraction than humans do. Most mammals with a panniculus carnosus have a plane of low resistance between the musculoskeletal tissue and the skin, which allows maximum skin mobility and contraction. Therefore, they do not have any excessive scars [2]. In humans, wound contraction is the greatest in the trunk and external genitalia, but the least in the extremities [2,7]; this is probably due to skin mobility. Thus, wound contraction in adult human extremities (especially the hand and foot) can produce hypertrophic scars and/or scar contracture.

In premature infants, however, the skin is still thin, reddish, and wrinkled with a small amount of subcutaneous fat due to immaturity [8]. Thus, the skin has less tensile strength and greater degree of laxity and mobility. In this study, closure of the defects was mainly dependent on wound contraction due to their histophysiological properties even in the extensive fullthickness defects of the dorsum of the hand and foot where the degree of contraction is relatively low in the adult. 
Inflammation and granulation tissue formation play key roles in adult wound healing, but not in that of an early fetus [3-10] because the amount of inflammation strongly correlates with the amount of scar formation [6]. Most of the inflammationinducing or -accelerating factors arise from the external environment ( $\mathrm{pH}$, bacteria, chemicals, dryness, and foreign bodies, etc.) or internal environment (blood cells and plasma). The fetal skin grafts heal wounds with scar formation after transplantation onto the skin of adult mice exposed to the air (external environment) [8]. After 24 weeks of gestation, human fetal amniotic fluid (external environment) does not reflect fetal plasma (internal environment) because of urine accumulation and reduced diffusion of the fetal plasma [1]. In late gestation, it is after a transition period, after 24 weeks when follicular keratinization is completed [14], that cutaneous wounds heal with scar formation $[5,7]$. Although the fetal environment alone cannot induce scarless healing in adult skin [10], these results suggest that scarless repair appears to depend on the external environment to some extent. Thus, our concept of local wound management is to create an optimal external environment for the wound.

In the current study, most of the full-thickness skin defects in the premature infants, who received both initial and continuous wound management, had no or minimal inflammation or granulation tissue formation, which resulted in pinpoint or linear scars. The minimally formed granulation tissue did not show a beefy-red appearance but was slightly protruded with a shiny surface despite of no bacterial growth in the wound culture. It was also not adhered to the marginal skin until wound closure. In contrast, there was an inflammatory response around the wound in the case of delayed initial treatment or discontinued wound management. Consequently, granulation tissue increased, gradually turned to a beefy-red appearance, and adhered to the wound margin during wound contraction. After epithelialization, this led to the appearance of a hypertrophic scar. Thus, we guessed that more granulation tissue, especially in a beefy-red appearance, probably inhibits wound contraction due to marginal adhesion and may result in an unsatisfactory round scar formation. In other words, reduction of the inflammatory response might play an important role in inhibiting granulation tissue and scar formation similar to fetal wound healing.

Local wound management in premature infants optimizes healing by maintaining a physiologic local wound environment, which is characterized by adequate moisture, normal body temperature, bacterial balance, and neutral to mildly acidic $\mathrm{pH}$ [12]. The moisture must be kept in balance to prevent both desiccation and maceration of the wound and surrounding skin [12]. We performed extensive local treatment of the whole area including the adjacent region and defect site using oil- and saline-moistened dressings. This was to protect and soften the wound and the surrounding skin. The 12 cases, which underwent proper treatment, were closed mainly by wound contraction, resulting in a satisfactory appearance of the pinpoint or linear scars without any significant functional deformity. In addition, the direction of wound contraction was usually vertical to the RSTL. Consequently, the linear scars were situated parallel to the RSTL. Furthermore, contracture was mild even in the extensive defects, which extended over two-thirds of the longitudinal length of the dorsum of the hand or foot. This suggests that enhancement of regional skin mobility is important for minimizing external scarring and for promoting wound contraction and closure. Thus, local treatment may need to be performed extensively up to the adjacent regions for the defect to heal with a satisfactory outcome until the scar is stabilized.

The total number of scars was inversely related to the gestational age and directly related to the duration of intensive care due to the significant lesions caused by the multiple invasive procedures for their survival [15]. To reduce the frequency and severity of skin damage, a more careful process of wound closure is needed. We expect that intensive wound management for moisturization and reduction of the inflammatory response could help enable the successful treatment of other defects of premature infants and extravasation wounds. This concept may also be applied to general wound management in adults.

This study had limitations, including the lack of an animal model research basisand a quantitative biochemical study. The quantitative extent of wound contraction in premature infants compared with those of adults remains somewhat unclear. Therefore, more studies are needed to reveal more precisely the wound healing process of premature infants.

\section{REFERENCES}

1. Cunningham FG, Leveno KJ, Bloom SL, et al. Fetal growth and development. In: Cunningham FG, Leveno KJ, Hauth JC, editors. Williams obstetrics. 22nd ed. Philadelphia: McGraw-Hill; 2005. p. 91-120.

2. Stoll BJ, Adams-Chapman I. The high-risk infant. In: Kliegman RM, Behrman RE, Jenson HB, et al., editors. Nelson textbook of pediatrics. 18th ed. Philadelphia: Saunders Elsevier; 2007.p. 698-711.

3. Broughton G 2nd, Janis JE, Attinger CE. Wound healing: an overview. Plast Reconstr Surg 2006;117:1e-S-32e-S.

4. Li J, Chen J, Kirsner R. Pathophysiology of acute wound healing. Clin Dermatol 2007;25:9-18.

5. Lorenz HP, Longaker MT. Wound healing: repair biology and wound and scar treatment. In: Mathes SJ, Hentz VR, 
editors. Plastic surgery. 2nd ed. Philadelphia: Saunders Elsevier; 2006. p. 209-34.

6. Ferguson MW, O’Kane S. Scar-free healing: from embryonic mechanisms to adult therapeutic intervention. Philos Trans R Soc Lond B Biol Sci 2004;359:839-50.

7. Dang C, Ting K, Soo C, et al. Fetal wound healing current perspectives. Clin Plast Surg 2003;30:13-23.

8. Longaker MT, Adzick NS. The biology of fetal wound healing: a review. Plast Reconstr Surg 1991;87:788-98.

9. Lorenz HP, Longaker MT, Perkocha LA, et al. Scarless wound repair: a human fetal skin model. Development 1992;114:253-9.

10. Longaker MT, Whitby DJ, Ferguson MW, et al. Adult skin wounds in the fetal environment heal with scar formation. Ann Surg 1994;219:65-72.

11. Afsar FS. Skin care for preterm and term neonates. Clin Exp Dermatol 2009;34:855-8.

12. Fox MD. Wound care in the neonatal intensive care unit. Neonatal Netw 2011;30:291-303.

13. Fluhr JW, Darlenski R, Taieb A, et al. Functional skin adaptation in infancy-almost complete but not fully competent. Exp Dermatol 2010;19:483-92.

14. Lane AT. Human fetal skin development. Pediatr Dermatol 1986;3:487-91.

15. Cartlidge PH, Fox PE, Rutter N. The scars of newborn intensive care. Early Hum Dev 1990;21:1-10. 


\section{Discussion}

\section{Prognosis of Full Thickness Skin Defects in Preterm Infants}

Seok-Chan Eun

Department of Plastic Surgery, Seoul National University Bundang Hospital, Seoul National University College of Medicine, Seongnam, Korea

The authors have written a prognosis of full-thickness skin defects in premature infants. The authors begin by dressing 14 cases of full-thickness skin defects due to extravasation of total parenteral nutrition. The authors described the dressing method, wound healing, and final scar. The timing of extravasation was 14 to 50 days after birth, and the gestational ages at birth ranged from 28 weeks and 4 days to 35 weeks and 6 days. This is the earliest clinical study of a premature infant wound healing process. Fetal wound healing is characterized by minimal inflammation and scarless repair. Premature infants are not completely the same fetuses, but premature infants in the early period after birth undoubtedly have characteristics in common with fetuses and, most relevantly to the present discussion, can heal without scarring.

Despite some important differences, the authors stress that intensive wound management for moisturization and reduction of an inflammatory response could help in the successful treatment of skin defects and lead to scarless repair. The biological phenomenon of scarless wound healing in the fetal period is not the same during the whole gestational period, but it would be the same in the postfetal wound healing process [1]. There is a developmentally regulated threshold for scarless healing based on both gestational age and the extent of injury [2]. The late-gestation fetus has been proven to heal with fibrosis and scarring [3]. Wound size also affects the degree of scarless repair [4]. This transition phenomenon has also been demonstrated in fetal sheep [5], rodents [6], and monkeys [7]. No one has been able to determine which factors have more influence on a final scar, and further studies are needed.

It seems as we understand more about scarless repair, we understand how much more we need to learn about the process. In the future, however, advances in technology might help us elucidate the mechanisms behind the wound healing process. More extensive research must be conducted to further define the integral process and the unknown aspects of fetal and scarless wound healing. It can be argued that well-intentioned clinicians, researchers, biologists, technicians, and industry personnel need to come together to better identify other mechanisms and principles in this rapidly developing subfield.

It is well known that human fetal wound healing research is practically impossible to conduct due to the bioethical issues. A premature infant is in a transitional stage between the fetus and adult stage, and thus has characteristics of both of them. From the prospective view, the wound healing process of premature infants could serve as a good surrogate for a scarless adult wound healing model.

\section{REFERENCES}

1. Bullard KM, Longaker MT, Lorenz HP. Fetal wound healing: current biology. World J Surg 2003;27:54-61.

2. Longaker MT, Peled ZM, Chang J, et al. Fetal wound healing: progress report and future directions. Surgery 2001;130: 785-7.

3. Larson BJ, Longaker MT, Lorenz HP. Scarless fetal wound healing: a basic science review. Plast Reconstr Surg 2010;126: 1172-80.

4. Cass DL, Bullard KM, Sylvester KG, et al. Wound size and gestational age modulate scar formation in fetal wound repair.J Pediatr Surg 1997;32:411-5.

5. Longaker MT, Whitby DJ, Adzick NS, et al. Studies in fetal wound healing, VI. Second and early third trimester fetal wounds demonstrate rapid collagen deposition without scar formation.J Pediatr Surg 1990;25:63-8.

6. Ihara S, Motobayashi Y, Nagao E, et al. Ontogenetic transition of wound healing pattern in rat skin occurring at the fetal stage. Development 1990;110:671-80.

7. Lorenz HP, Whitby DJ, Longaker MT, et al. Fetal wound healing. The ontogeny of scar formation in the non-human primate. Ann Surg 1993;217:391-6.

\section{Correspondence: Seok-Chan Eun}

Department of Plastic Surgery, Seoul National University Bundang Hospital, Seoul National University College of Medicine, 82 Gumi-ro 173beon-gil, Bundang-gu, Seongnam 463-707, Korea

Tel: +82-31-787-7223, Fax: +82-31-787-4055, E-mail: sceun@snubh.org

No potential conflict of interest relevant to this article was reported.

Received: 7 Aug 2012 • Revised: 8 Aug 2012 • Accepted: 9 Aug 2012 pISSN: 2234-6163 • elSSN: 2234-6171

http://dx.doi.org/10.5999/aps.2012.39.5.469 • Arch Plast Surg 2012;39:469

Copyright $(\odot 2012$ The Korean Society of Plastic and Reconstructive Surgeons

This is an Open Access article distributed under the terms of the Creative Commons Attribution Non-Commercial License (http://creativecommons.org/

licenses/by-nc/3.0/) which permits unrestricted non-commercial use, distribution, and reproduction in any medium, provided the original work is properly cited. I www.e-aps.org 